\title{
Revisión de algunas variables relevantes en el establecimiento de metas deportivas
}

\author{
Jaime Díaz-Ocejo ${ }^{1}$ y Juan Antonio Mora-Merida ${ }^{2 *}$ \\ 1 ASPIRE, Academy for Sport Excellence, Doha, Qatar \\ ${ }^{2}$ Departamento de Psicología Básica, Facultad de Psicología. Universidad de Málaga, España
}

\begin{abstract}
Resumen: El propósito del presente trabajo es examinar la influencia de algunas variables relevantes en el proceso de establecimiento de metas, como son la proximidad, la especificidad, la dificultad y la efectividad de las mismas, a través de la revisión de la literatura científica al respecto. Para ello, se siguen las directrices señaladas por autores destacados en este ámbito de estudio. Los resultados de la revisión no revelan posturas concluyentes en relación a la influencia de la proximidad, la especificidad y la dificultad de las metas. Contrariamente, se encuentran evidencias más consistentes a favor de la efectividad del empleo de esta estrategia psicológica como herramienta para la mejora del rendimiento en distintas tareas motoras.

Palabras clave: Metas; proximidad; especificidad; dificultad; efectividad.
\end{abstract}

\section{Introducción}

Al igual que ocurre en otros aspectos de la vida, las conductas humanas que persisten a lo largo del tiempo en el ámbito de la actividad física y deportiva, lo harán, posiblemente, a pesar del hecho de que las recompensas externas sean mínimas o inexistentes (Díaz-Ocejo, 2006). El establecimiento de metas puede suponer una herramienta motivacional útil para la consecución de logros personales, de grupo, de rendimiento, del propio proceso o para incrementar los beneficios en nuestra salud. Igualmente, si bien el deporte ha evolucionado históricamente para dotarse a sí mismo de metas claras, ello no significa que automáticamente uno descubra lo que tiene que hacer simplemente con participar, ya que es necesario establecer pautas precisas que servirán como guía durante todo el proceso (Jackson y Csikszentmihalyi, 2002; Dosil, 2004).

Con un enfoque que podríamos denominar como clásico, el establecimiento de metas siempre ha estado presente en la Psicología del Deporte, sea bajo el prisma del aprendizaje de la táctica deportiva (Riera, 1994); o bien en la construcción de instrumentos que nos permitieran medir y evaluar posteriormente las metas (Hernández-Mendo y Ramos-Pollán, 1995); así como en cómo el deportista domina las tareas en las que se halla inmerso (Riera, 1997). Con un enfoque más de intervención, el establecimiento de metas está, implícito o explícito, en los planes de entrenamiento psicológico que realizamos con los más diversos deportes (González y Garcés de los Fayos, 2009).

Algunos han remarcado, igualmente, la gran importancia del establecimiento de metas en la cohesión y cooperación de los equipos deportivos (Olmedilla, Ortega, Almeida, Lameiras, Villalonga, Sousa, Torregrosa, Cruz y García-Mas, 2011), así como pueden ser vistas como un magnífico predictor de la marcha del equipo, con especial incidencia en los deportistas

* Dirección para correspondencia [Correspondence address]: Juan Antonio Mora-Mérida, Departamento de Psicología Básica, Facultad de Psicología, Universidad de Málaga. 29071. Málaga (España). E-mail: mora_merida@uma.es

\begin{abstract}
Title: Literature Review on Some Relevant Variables in Goal Setting in Sport.

Abstract: The purpose of this paper is to examine the influence of some relevant variables in the goal setting process, such as the proximity, specificity, difficulty and effectiveness, throughout the literature review. Accordingly, we follow the guidelines outlined by relevant authors in this topic. Results are not conclusive with regards to the proximity, specificity and difficulty in goal setting. Contrarily, it is found a more consistent evidence in favor of the effectiveness in the use of this psychological strategy as a tool to enhance motor skills performance.

Key words: Goals; proximity; specificity; difficulty; effectiveness.
\end{abstract}

más jóvenes (Moreno, Cervelló y González, 2010). Igualmente, en contextos alejados del deporte profesional, el establecimiento de metas puede ser saludado como un indicador que nos refuerza los perfiles motivacionales de los usuarios de instalaciones deportivas, en contextos amplios (Sicilia, Aguila, Muyor, Orta y Moreno, 2009).

Las metas por sí mismas no garantizan la consecución del éxito, sino que incorporadas sistemáticamente en la planificación global del deportista, permiten su establecimiento, planificación, dirigir la atención hacia las mismas y su evaluación para una mejor organización de sus conductas y pensamientos (Gano-Overway, 2008, Burton, Naylor y Holiday, 2001; Vealey, 2007; Mora, García, Toro y Zarco, 2000). Una de las aproximaciones conceptuales más referidas en la literatura en Psicología del Deporte sobre el establecimiento de metas, es la aportada por Locke, Shaw, Saari y Latham (1981) quienes la definen como: "El logro de un nivel específico de dominio de una tarea, generalmente dentro de un tiempo especificado". (p. 145).

Esta definición ya se orienta hacia el que sería, posteriormente, uno de los elementos más investigados en el establecimiento de metas en el deporte, como es la especificidad de las mismas y la inclusión de la variable temporal (proximidad de la misma), elemento que ha dado cuerpo a un número elevado de investigaciones (Gould, 1991). Esta aproximación temporal sobre el establecimiento de metas daría como resultado la clasificación de metas a corto y largo plazo, aunque posteriormente cobraría también relevancia el establecimiento de metas intermedias, como enlace entre las dos anteriores. Poco después, McClements (1982) establecería distinciones entre los posibles tipos de metas en base a la subjetividad u objetividad de las mismas, así, propone que las metas puedan ser metas subjetivas (aquellas que no presentan objetivos específicos), metas objetivas generales (las que se centran en un objetivo no muy concreto, por ejemplo ganar un campeonato), y las metas objetivas específicas (aquellas que son cuantificables y por lo tanto medibles, como los tiempos concretos en series de natación, kilos levantados en sesiones de entrenamiento de pesas, etc.). 
Martens, Christina, Harvey y Sharkey (1981) y Burton (1983, 1984), establecieron distinciones entre las metas de resultado, que son las orientadas a la consecución de la victoria o récord en la competición, y metas de rendimiento, que se refieren a las mejoras relativas a la propia ejecución que se ha tenido anteriormente (se puede quedar segundo en un campeonato del mundo, pero mejorar la marca personal o centrarse en un punto concreto del gesto técnico para mejorar el rendimiento). En el ámbito genérico de la actividad física y deportiva, autores como Hardy, Jones y Gould (1996), Burton et. al., (2001) o Cox (2002) han completado esta clasificación sobre las metas y las han ampliado a metas de resultado, metas de rendimiento y metas de realización o de proceso. Siguiendo a Dosil (2004), las metas de realización o de proceso se distinguen de las anteriores en cuanto a que orientan al deportista a centrarse en las conductas y pensamientos relativas a la consecución de las metas de proceso y de rendimiento. En ese sentido, la relevancia de cada una de este tipo de metas dependerá de factores como el momento en la temporada o la motivación del deportista (Dosil, 2004).

El propósito del presente trabajo es examinar la influencia de algunas variables relevantes en el proceso de establecimiento de metas, como son la proximidad, la especificidad, la dificultad y la efectividad de las mismas, a través de una revisión de la literatura científica al respecto. Para ello, se siguen las directrices señaladas por autores destacados en este ámbito de estudio como son Locke y Latham (1985), Burton et al., (2001) y Gould (1991). En primer lugar, se aborda brevemente la conexión entre establecimiento de metas y la actividad física y deportiva, con el objeto de considerar los fundamentos originales de esta estrategia psicológica. En segundo lugar, se realiza una revisión de las variables mencionadas y su influencia en el proceso de establecimiento de metas y, finalmente, se comentan algunas conclusiones derivadas de la presente revisión.

\section{Establecimiento de metas y la actividad física y el deporte}

El concepto de establecimiento de metas puede ser comprendido a partir de dos corrientes tradicionales de investigación; la academicista y la organizacional. De acuerdo con Ryan (1970), la corriente académica, que se remonta a principios de los años sesenta, se centra en los conceptos asociados de intención, establecimiento de la tarea y el nivel de aspiración. Por otro lado, la obra de Taylor (1967) The Principles of Scientific Management, abriría la línea de investigación desde una perspectiva organizacional, introduciendo el elemento de la tarea (definida como misión o meta) como punto de referencia para gestionar los objetivos que tenían los trabajadores día a día. Influenciado por ambas corrientes, es fundamentalmente a partir de los trabajos de Locke (1966, 1968) con su Teoría del Establecimiento de Metas, donde se inicia una base de investigación que supuso que la teoría del establecimiento de metas demostrara, hasta la fecha, más va- lidez científica que cualquier otra teoría o modelo a cerca de la motivación en el ámbito del trabajo (Pinder, 1984). Locke (1966, 1968) afirmó que las metas específicas, desafiantes y difíciles, llevaban a mayores niveles de ejecución de la tarea que las metas fáciles, inconcretas o inexistentes, así, el aspecto más investigado a cerca de su teoría ha sido la dificultad o especificidad de la meta y su influencia en la ejecución. Las previsiones relativas a la extrapolación de los hallazgos encontrados en el ámbito organizacional e industrial con respecto sus las posibilidades en la Psicología del Deporte, no se han correspondido con lo evidenciado por la literatura científica en ese campo hasta fechas recientes (Weinberg, 1995). Quizá, tal y como comentan Hall y Kerr (2001), la diferencia entre ambos contextos estribe en la variable elección, puesto que los trabajadores tienen que ir al trabajo reduciéndose sus posibilidades de elegir. No obstante, los autores sí consiguieron, a partir de este trabajo, llamar la atención y fomentar las investigaciones en esta estrategia que, eventualmente, sí ha dado resultados prometedores. Locke y Latham (1985), presentaron diez presupuestos específicos acerca de cómo las metas pueden funcionar en el contexto deportivo, a partir de las experiencias obtenidas en el ámbito organizacional, tal como puede apreciarse en la Tabla 1:

Tabla 1. Aplicaciones del establecimiento de objetivos en el deporte. Adaptado de Locke y Latham (1985, p. 212).

1)Las metas específicas regularán la acción con más precisión que las metas generales.

2) Para las metas cuantitativas (específicas), cuanto más alta sea la meta, mejor es la ejecución, teniendo en cuenta la habilidad y el compromiso suficientes.

3)Las metas específicas y difíciles, llevaran a una mejor ejecución que las metas de hazlo lo mejor que puedas o la ausencia de metas.

4)Utilizar metas a corto y a largo plazo, produce mejores resultados que utilizar solamente metas a largo plazo.

5)Las metas afectan a la ejecución, movilizando el esfuerzo, incrementando la persistencia y motivando la búsqueda de estrategias adecuadas.

6)El establecimiento de metas será más efectivo cuando se da una retroalimentación que muestre el progreso en relación a la meta.

7)Con metas difíciles, cuanto mayor es el compromiso, mejor es el rendimiento.

8)El compromiso puede estimularse pidiendo al individuo que acepte la meta, mostrando apoyo, permitiendo la participación en el establecimiento de la meta, en el entrenamiento, en la selección de los incentivos y recompensas.

9)La ejecución de la meta se verá facilitada mediante un plan de acción o estrategia convergente, especialmente cuando la tarea es compleja o a largo plazo.

10) La competición mejorará la ejecución pues conduce a establecer objetivos más altos y a aumentar el grado de compromiso.

A pesar de que Locke y Latham (1985) sugirieron que las tareas desarrolladas en el ámbito de las organizaciones y en el laboratorio tienen mucho en común con las actividades deportivas, ya que ambas implican actividades mentales y físicas dirigidas hacia un fin objetivo, es sorprendente que este aspecto haya sido poco abordado en la literatura psi- 
cológica de orientación deportiva (Gould, 1991). En la presente revisión sobre la influencia de algunas variables y su desarrollo en las investigaciones que se han llevado a cabo ligadas al establecimiento de metas en la actividad física y el deporte, nos apoyamos parcialmente en la estructura presentada por autores como Locke y Latham (1985), Burton et al., (2001) o Gould (1991). Estos autores destacan la especificidad, la dificultad, la proximidad en el tiempo y la efectividad de los objetivos, entre otras, como variables influyentes en el establecimiento de metas.

\section{La especificidad de las metas}

Entre los primeros autores en hacer referencia a la especificidad de las metas en el contexto de la actividad física, se encuentran Locke y Bryan (1966). Estos autores examinaron los efectos de una tarea de coordinación psicomotora compleja con estudiantes universitarios, que fueron asignados a dos grupos; uno con metas establecidas por los experimentadores, y el grupo control a quien se les dijo que lo hicieran lo mejor posible. Los resultados apuntaron a una mejor ejecución en el grupo al que se le habían asignado metas específicas. Barnett y Stanicek (1979), por su parte, investigaron los efectos que el establecimiento de metas tendría en un grupo de 40 alumnos universitarios que aprendían tiro con arco. Para ello, los sujetos fueron divididos en dos condiciones, una a la que se le indicó que establecieran las metas numérica y verbalmente por ellos mismos (condición experimental), y otra a quien no se le propuso establecer ninguna meta (condición control). Los resultados encontrados revelaron diferencias significativas a favor del grupo experimental, por lo que el establecimiento de metas había tenido efectos favorables. Davis y Spennewyn (1983) examinaron cómo de específicos, próximos y difíciles eran los objetivos que se planteaban un grupo de 38 deportistas (nadadores y corredores de campo a través) de nivel universitario. Los resultados mostraron claramente que los sujetos relacionaban, a través del empleo de un cuestionario de respuestas abiertas, que el éxito deportivo estaba relacionado con la especificidad de las metas.

Sin embargo, no todas las primeras investigaciones orientadas a la especificidad de las metas obtuvieron resultados favorables. Así, Hollingsworth (1975) y Barnett (1977), en una tarea de malabarismos; Hall, Weinberg y Jackson (1983), a través del empleo de un circuito de entrenamiento; o Stitcher, Weinberg y Jackson (1983) en una tarea de levantamiento de pesas, no hallaron diferencias significativas entre ambos grupos experimental y control. En efecto, Hollingsworth (1975) investigó a través de una tarea de malabarismos con 90 estudiantes de Educación Secundaria los efectos de la especificidad de la meta. Un grupo fue asignado a las metas de rendimiento, otro a la condición hažlo lo mejor que puedas, y un tercer grupo como control. Los resultados obtenidos no mostraron diferencias significativas entre los tres grupos. Igualmente, Barnett (1977) recurrió en su trabajo a 93 alumnas de Educación Secundaria quienes fueron distribuidas en cinco condiciones diferentes también ante una prueba de malabarismos; a) un grupo con metas específicas, b) un grupo en el que las alumnas se apoyaban unas a otras, c) otro grupo que incluía una charla previa del profesor y además con metas específicas, d) un grupo en el que el profesor apoyaba a los alumnos y, c) un grupo de control. Los resultados no revelaron ninguna diferencia entre los grupos. A la vista de estas evidencias, Hollingsworth (1975) y Barnett (1977) concluyeron que, debido a la naturaleza de la tarea, los alumnos podían establecer sus propias metas en base a los resultados que iban obteniendo.

Por su parte, Weinberg, Bruya y Jackson (1985) investigaron esta hipótesis con estudiantes universitarios de una clase de acondicionamiento físico a través de una prueba de abdominales. Tras establecer una línea base de rendimiento para emparejar a los sujetos, estos fueron asignados aleatoriamente a una de las cuatro condiciones de establecimiento de metas. Para tres de las condiciones, los sujetos tenían una meta específica difícil, mientras que para la cuarta condición la premisa era hazlo lo mejor que puedas. Tras un período experimental de cinco semanas, no se encontraron diferencias significativas entre los tres grupos de meta específica dificil y la condición control (hazlo lo mejor que puedas). Posteriormente, Weinberg, Bruya, Jackson y Garland (1987) y Garland, Weinberg, Bruya y Jackson (1988) replicarían ese trabajo en condiciones similares de acondicionamiento físico y también a través de una prueba de abdominales de tres minutos de duración. Tras un período de cinco semanas, los resultados apuntaron, de nuevo, a ninguna diferencia entre los sujetos asignados a grupos de establecimiento de metas específicas y difíciles con respecto a la condición de asignación de metas inespecíficas.

Boyce (1994) examinó los efectos de las metas específicas en un grupo de 30 tiradores de pistola veteranos. Los sujetos fueron distribuidos en un grupo de establecimiento de meta y otro a una condición de hazlo lo mejor que puedas. Los resultados no sólo no evidenciaron diferencias significativas entre ambos grupos sino que, inesperadamente, los sujetos del grupo hazlo lo mejor que puedas obtuvieron resultados ligeramente superiores a los del grupo experimental. Boyce (1994) concluyó que esos resultados se debían a que parte de los tiradores del grupo control habían establecido sus propias metas durante el período de 4 semanas que duró la investigación. Otros resultados que llaman la atención en este sentido serían los encontrados por Kenan y Lord (1988), cuyos sujetos sin metas específicas generalmente evaluaron su rendimiento de un modo más positivo que el grupo al que le habían sido asignadas metas específicas y difíciles. Mento, Locke y Klein (1992) demostraron que los sujetos en la condición hazlo lo mejor que puedas mostraron un nivel superior de satisfacción que los que habían sido asignados a la condición de metas difíciles y específicas. Otros autores como Correa, de Souza y Santos (2006), no encontraron diferencias significativas en su trabajo sobre la adquisición de un gesto técnico en voleibol, entre tres grupos experimentales (metas genera- 
les, metas específicas a largo plazo, metas específicas a corto plazo), y una condición control.

Como puede apreciarse, los resultados a cerca de la relación existente entre la especificidad de la meta en relación al rendimiento en tareas de fuerza-resistencia, no parecen ser concluyentes. Quizá, sería más sensato manifestarse en este sentido de un modo prudente, como lo hacen Weinberg y Gould (2003): "Las metas específicas, en comparación a las de -hazlo lo mejor que puedas-, son más efectivas para producir cambios comportamentales" (p.336). Es decir, la aplicación de este tipo de metas puede favorecer la mejora del rendimiento modificando la conducta del deportista, a través de una mejor disposición y orientación hacia los entrenamientos y competiciones. Posiblemente, las metas específicas podrían entonces estar relacionadas de algún modo con las metas de realización o proceso, y no directamente con las de rendimiento o resultado.

\section{La dificultad de las metas}

Otros estudios en el contexto del establecimiento de metas en la actividad física y el deporte, se han desarrollado a partir de la hipótesis de Locke y Latham (1985) de que la dificultad de la meta sería más eficaz en la mejora de la ejecución que las metas fáciles o de hazlo lo mejor que puedas. Botterill (1977) realizó una de las primeras investigaciones de establecimiento de metas en un grupo de jugadores de hockey. Los resultados revelaron que el establecimiento de metas difíciles conllevaba mejoras en el rendimiento de la tarea en comparación con las metas fáciles. Poco después, el mismo Botterill (1979) encontró que los sujetos a quienes se les asignó una meta difícil y específica obtenían mejores resultados que aquellos a quienes se les dijo que lo hicieran lo mejor que pudieran. Este autor llevó a cabo una tarea de resistencia física con, entre otras, varias combinaciones de dificultad. Sin embargo, según Weinberg (1995), algunas carencias metodológicas como establecer quien fijaba las metas, sujeto o experimentador, grupal o individual, llevaron a resultados confusos en ambos estudios.

En relación a la dificultad de la meta con la ejecución, Garland (1983) cuestionó el principio de la posibilidad del logro de la meta, tal y como sugieren Locke y Latham (1985). En efecto, este autor subrayó que los distintos estudios de laboratorio habían llevado a lo que él denominó relaciones monotónicamente positivas entre la dificultad de la meta y la ejecución. Es decir, en distintos experimentos se observaba que los sujetos a quienes se asignaba una meta difícil o fuera de su alcance, fracasaban sucesivamente, aunque ello no empeoraba la ejecución o rebajaba su motivación. Del mismo modo, siguiendo a Garland (1985), un descenso en la motivación a consecuencia de repetidos fracasos en el rendimiento de la tarea, produciría algún tipo de relación de U invertida entre la dificultad de la meta y la ejecución, aunque este no parece ser el caso habitual. Esta orientación novedosa en el devenir de los trabajos orientados a la viabilidad de la me- ta en relación a su dificultad, propuesta por Garland (1985), atrajo la atención de autores como Weinberg et al. (1987).

En el primer experimento, estos investigadores contaron con 200 sujetos para una prueba de abdominales durante tres minutos. Las condiciones a las que fueron repartidos (meta fácil, meta moderadamente difícil y meta muy difícil) dependían de los resultados obtenidos en un test previo como línea base, también de abdominales en tres minutos. Tras un entrenamiento de cinco semanas, los resultados no mostraron diferencias significativas entre la ejecución de los tres grupos. Sin embargo, la mayoría de los sujetos mejoraron al final de las cinco semanas. Lo que apoyaba la teoría de Garland (1985) a partir de la cual la dificultad de la de la meta no empeoraría el rendimiento.

En un segundo experimento, Weinberg et al. (1987), asignaron a los sujetos a tres grupos, de los cuales dos afrontarían la viabilidad de la meta con más dificultad. Un primer grupo tendría una meta muy difícil (mejorar en 40 abdominales), otro grupo una meta altamente improbable (mejorar en 60 abdominales), y un tercer grupo control de meta no específica. Los resultados, de nuevo, no reflejaron ninguna diferencia significativa entre los tres grupos y tampoco se dio ningún decrecimiento en el rendimiento, aunque ningún sujeto de la condición de 60 abdominales como meta, logró alcanzarla. Los resultados de ambos experimentos apoyaron la hipótesis de Garland (1985), ya que la relación entre la dificultad de la meta no perjudica la ejecución o rendimiento. Aunque la mayoría de los trabajos llevados a cabo sobre el establecimiento de metas han sido dirigidos hacia la verificación de los postulados de Locke $(1966,1968)$ a partir de la relación entre metas y ejecución, posteriormente, Garland (1985) ha propuesto un nuevo modelo de estudio a este respecto. En ese sentido, este autor define la meta individual como "una imagen de un nivel futuro de ejecución que el individuo desea lograr" (p. 347). De esta manera, nos presenta la Teoría de la Mediación Cognitiva como alternativa para la investigación científica en este ámbito. Básicamente, esta Teoría apunta a que los individuos desarrollan expectativas subjetivas para alcanzar muchos niveles diferentes de ejecución (que Garland denomina expectativa de ejecución) y no únicamente el nivel representado por una meta en la tarea (que él denomina valencia de ejecución). Este autor establece similitudes entre su Teoría y la Teoría de Auto-eficacia de Bandura (1977), tal como se aprecia en la Tabla 2.

Tabla 2. Aspectos fundamentales diferenciadores entre la perspectiva de Locke y Latham (1985) y la de Garland (1985) acerca de la dificultad de las metas.

Locke y Latham (1985) Garland (1985)

Postulado basado en las carac- Teoría de la Mediación Cognitiva, terísticas objetivas de la difi- donde se tiene en consideración la cultad de la meta fijada para el expectativa subjetiva del individuo individuo respecto a la meta

En relación a la dificultad de metas y el rendimiento en el deporte de élite, es relevante mencionar los resultados obtenidos por Weinberg, Burton, Yukelson y Weingand (2000), 
en su revisión sobre los hábitos de establecimiento de metas con deportistas olímpicos. Estos autores encontraron que establecer metas a un nivel algo superior a sus mejores rendimientos era el más preferido por 185 hombres y 143 mujeres que competían a ese nivel deportivo. Por lo que, atendiendo a estos resultados y los derivados de los trabajos comentados anteriormente, parece ser que establecer metas por encima de lo que se está rindiendo en esos momentos tiene consecuencias positivas para la mejora del rendimiento deportivo, lo cual, de nuevo, nos llevaría a tener en consideración la sugerencia inicial de Locke $(1966,1968)$. Por otra parte, en un estudio realizado en el ámbito escolar, Bar-Eli, Tenenbaum, Pie, Btesh y Almog (2007), encontraron que los sujetos en la condición asignada a metas realistas y difíciles mostraron una mejora más sustancial, en relación a los grupos de metas específicas y a los de no asignación de metas.

A partir de la complejidad de establecer metas en cuanto a su dificultad, Orlick (2000) ha sugerido que la optimización de la dificultad de meta puede ir en la línea de; a) recomendar a los deportistas que se marquen metas de ensueño (metas muy difíciles, pero sólo si estos están rindiendo al máximo nivel posible, b) establecer metas realistas o de dificultad moderada y, c) metas de auto-aceptación (las metas de nivel más bajo en las que el/la deportista aún se siente satisfecho). Más recientemente, Bueno, Weinberg, Fernández-Castro y Capdevilla (2009) han señalado que el no alcanzar las metas establecidas puede suponer una amenaza para el deportista. Ello provocaría una percepción de indefensión en el mismo, sobre todo si las metas son muy difíciles, por lo que es recomendable mantener niveles adecuados de auto-eficacia para controlar este tipo de situaciones.

\section{La proximidad de las metas}

En muchas ocasiones, cuando se les pide a los deportistas que describan sus metas, estos las identifican como metas a largo plazo (Gould, 1991). En efecto, los deportistas involucrados en la competición a menudo se marcan como reto ganar un campeonato, batir un record o mejorar su marca personal. Sin embargo, algunos psicólogos del deporte en la década de los ochenta, como Bell (1983), Carron (1984), O’Block o Evans (1984), ya destacaron la importancia de establecer objetivos intermedios o inmediatos para la consecución de la meta final. Por lo tanto, estamos ante otra de las sugerencias realizadas por Locke y Latham (1985) que también ha generado un sustancioso cuerpo de investigaciones al respecto. Estos autores postularon que el establecimiento de metas a corto plazo junto con las metas a largo plazo, produciría mejores resultados que simplemente establecer metas a largo plazo. En ese sentido, la motivación jugaría un papel importante debido a que los objetivos a largo plazo no fomentarían tanto la intensidad de esta variable. Así, Bandura (1982) comenta que las metas a corto plazo son fundamentales para mejorar la ejecución, ya que ofrecen una retroalimentación e incentivo inmediato, regulando el progreso del individuo. Similarmente, Carver y Scheier (1982), rela- cionan positivamente el establecimiento de metas a corto plazo, con un cambio conductual auto-regulado sustancial y duradero.

Para Kirschenbaum (1985), el mayor beneficio del empleo de las metas a corto plazo depende de la flexibilidad de las mismas. En su revisión, este autor concluyó que las metas rígidas (por ejemplo, hacer pesas todos los días) no tendrían efectos tan favorables como establecer metas flexibles (por ejemplo, hacer una sesión de pesas de 40 minutos cinco días a la semana). Hay que tener en cuenta que, en el ámbito del entrenamiento deportivo, cualquier lesión o imposibilidad de llevar a cabo el entrenamiento, puede derivar en sentimiento de impotencia al no poder cumplir con el objetivo rígido establecido. Del mismo modo, la Psicología del Deporte no ha presentado suficientes aportaciones a este respecto y los resultados obtenidos están lejos de ser concluyentes.

En el trabajo de Weinberg et al. (1985), los sujetos en una tarea de abdominales fueron asignados aleatoriamente a tres condiciones diferentes (a corto plazo, a largo plazo y a corto y largo plazo). A los sujetos incluidos en el grupo de meta a corto plazo, no se les hizo mención de meta a largo plazo, a los sujetos asignados a la condición meta a largo plazo, no se les indicó ninguna meta a corto plazo o semanal (el estudio tenía una duración de 5 semanas), y a los sujetos pertenecientes al grupo de metas a corto más a largo plazo, recibieron una combinación de instrucciones. Los resultados no mostraron diferencias significativas entre ninguna de las condiciones establecidas. A partir de las consideraciones expresadas por Kirschenbaum (1985), Hall y Byrne (1988) llevaron a cabo un estudio acerca de la proximidad de la meta, también con una tarea de abdominales, estableciendo modificaciones metodológicas. De ese modo, los sujetos fueron asignados aleatoriamente a cuatro condiciones; metas a largo plazo, metas a largo plazo más metas a corto plazo establecidas por el experimentador (no flexibles), metas a largo plazo más metas a corto plazo establecidas por el sujeto (más flexibles), y una condición control de metas no específicas. Los resultados no arrojaron diferencias significativas entre los grupos de metas establecidas por el experimentador y por los sujetos mismos. Sin embargo, todos los grupos obtuvieron mejores resultados que los obtenidos por la condición control. En ninguno de estos dos trabajos se aprecian diferencias significativas entre los grupos de metas establecidas a corto plazo con respecto a los de metas a largo plazo, en relación a la flexibilidad de la meta. Con lo que la conclusión establecida por Kirschenbaum (1985), no se vio respaldada experimentalmente.

Posteriormente, Boyce (1992) llevó a cabo otro trabajo con estudiantes universitarios, esta vez, en una tarea de tiro con carabina. Esta autora pretendía investigar los efectos de la proximidad de la meta en relación con la ejecución y en comparación con no establecer ninguna meta. Para ese propósito, contó con 111 hombres y 70 mujeres que fueron distribuidos aleatoriamente a uno de los siguientes grupos; una condición de metas a corto plazo, otra de metas a largo plazo, otra condición de metas a corto plazo más largo plazo 
y, finalmente, un grupo de hazlo lo mejor que puedas. Los resultados indicaron que existían diferencias significativas entre los tres grupos de establecimiento de metas específicas, en comparación con la condición hazlo lo mejor que puedas, en relación al rendimiento.

En efecto, en esta investigación se puso de manifiesto que establecer metas en distintos niveles de proximidad tenía efectos favorables para el rendimiento en la tarea. Sin embargo, no se informó de las posibles diferencias encontradas entre las distintas condiciones de proximidad de meta. A raíz de estos resultados obtenidos en la literatura científica, acerca de la proximidad de las metas, no se pueden establecer afirmaciones concluyentes. No obstante, algunos autores han insistido que centrarse únicamente en metas a largo plazo no mejoran el rendimiento (Kyllo y Landers, 1995), o que ambas, metas a corto y a largo plazo, son importantes cara a la consecución de altos logros deportivos (Kane, Baltes y Moss, 2001).

\section{La efectividad de las metas}

Siguiendo las aportaciones de Burton (1983) a este respecto, marcarse retos en los ambientes atléticos, guarda relación directa con niveles de ansiedad, motivación y confianza. Es decir, las metas muy difíciles o poco realistas centradas en el resultado, pueden influir en los niveles de esas tres variables (o alguna de ellas) en caso de no ser alcanzados. Esta situación puede llevar a efectos indeseados por parte del deportista derivando en un detrimento de su rendimiento. Burton (1983) sugiere como alternativa, tener en consideración los beneficios del establecimiento de metas de realización que, siendo aplicadas correctamente, pueden favorecer que el deportista se plantee metas más realistas y objetivas. De hecho, Burton (1983) llevó a cabo la primera investigación a través de un programa de entrenamiento en establecimiento de metas en natación.

El objetivo del estudio era examinar la efectividad que el mencionado programa tendría, no sólo en el rendimiento, sino también en distintas cogniciones como el nivel de autoconfianza, motivación y estados de ansiedad. El programa de establecimiento de metas tuvo una duración de cinco meses y se contó con nadadores de varias universidades y de ambos sexos. Los resultados revelaron que los nadadores que habían participado en el programa de entrenamiento aprendieron a focalizarse hacia el proceso de ejecución (meta de realización) de las metas más altas, y que los nadadores de mayor nivel de rendimiento lo mejoraron a la vez que mostraron cogniciones más favorables.

Estos resultados fueron avalados posteriormente (Burton, 1984) también con nadadores, donde se demostró que las metas correlacionaban positivamente con la ejecución y con los atributos cognitivos positivos. Anteriormente, Botterill (1977) ya había investigado a través de una tarea de resistencia la efectividad que el establecimiento de metas tendría en esa tarea. Al igual que venía ocurriendo con la literatura de la psicología general, los resultados de este estudio pusieron de manifiesto los beneficios de la dificultad y especificad de la meta, en contraposición a los efectos de las metas de tipo bazlo lo mejor que puedas. Igualmente, se destacó la importancia de la efectividad del establecimiento de meta grupal para la mejora del rendimiento individual. En relación a esta última evidencia sobre la importancia de las metas grupales y su efectividad sobre el rendimiento individual, Johnson, Morgantown, Ostrow, Perna y Etzel (1997) también encontraron resultados similares. En el trabajo de estos autores, realizado con jugadores de bolos noveles, se demostró que el hecho de establecer metas a nivel grupal beneficiaba el rendimiento de los jugadores a nivel individual bajo condiciones de elevada exigencia de rendimiento. Demostrándose los efectos de la variable grupal, sobre la individual.

Acerca de la efectividad del establecimiento de metas, y su influencia sobre el rendimiento y cognición, Burton (1989) llevó a cabo otra investigación con nadadores concluyendo que los deportistas que establecían metas altas obtenían mejoras en el rendimiento y además aprendían con efectividad a establecer sus propias metas. Igualmente, estos nadadores mostraron niveles más bajos de ansiedad, y mayores puntuaciones en auto-confianza que los del grupo control. Por su parte, Weinberg, Burton, Yukelson y Weigand (1993) llevaron a examen las percepciones de 357 hombres y 321 mujeres que competían en la liga de mayor nivel universitario en diferentes deportes, incluyendo la efectividad, entre otras, del establecimiento de metas. Prácticamente todos los deportistas empleaban algún tipo de establecimiento de objetivos de dificultad moderada a alta. Los resultados en relación a la efectividad de las metas apuntaron a que los efectos más importantes de la aplicación de esta estrategia eran mejorar el rendimiento, ganar y divertirse.

Curiosamente, las mujeres manifestaron establecer mayormente metas de realización en contraposición a los varones, quienes establecían metas de resultado con más asiduidad. De un modo semejante, Weinberg, Burke y Jackson (1997) investigaron este aspecto con 137 chicos y 87 chicas tenistas de edades comprendidas entre los 14 y los 18 años. Los resultados evidenciaron que los tres efectos más importantes en el establecimiento de metas, según los tenistas, eran mejorar el rendimiento, divertirse y ganar. Al igual que en el trabajo de Weinberg et al. (1993), los objetivos moderados a altos eran los preferidos por estos deportistas por sus mejores efectos cara a la competición.

En la posterior revisión de Kyllo y Landers (1995), encaminada a comprobar la efectividad del establecimiento de metas, también se puso de manifiesto sus efectos favorables para la mejora del rendimiento deportivo. Estos autores revisaron 36 trabajos realizados en el ámbito de la actividad física y el deporte, llegando a la conclusión de que el establecimiento de metas contribuye a la mejora del rendimiento deportivo al $0.34 \%$ de la desviación típica. Siendo los mejores efectos alcanzados a través del establecimiento de metas moderadas, difíciles o una combinación de metas a corto y a largo plazo. 
Uno de los escasos programas de entrenamiento específico en establecimiento de metas que aparece en la literatura científica, fue el llevado a cabo por Pierce y Burton (1998). Estos autores investigaron los efectos que este tipo de programas podrían tener en un grupo de 25 chicas gimnastas de entre 12 y 14 años de edad. Tras evaluar sus estilos de establecimiento de meta (orientado al fracaso, orientado al rendimiento u orientado al éxito), los resultados obtenidos apuntaron a una relación entre el estilo presentado por las gimnastas y sus resultados a lo largo de la temporada. Así, las deportistas orientadas hacia el establecimiento de metas para el fracaso, no llegaron a participar en uno de los aparatos de la competición, en todas las competiciones. Las gimnastas orientadas hacia el éxito deportivo, obtuvieron una ligera disminución en su rendimiento, mientras que las que mostraban un estilo focalizado hacia rendimiento durante las rutinas, obtuvieron una mejora significativa en sus ejecuciones. En una evaluación posterior al término de la temporada, las gimnastas que habían presentado un estilo de establecimiento de metas hacia el rendimiento, también fueron las que más favorablemente evaluaron el programa. Por lo tanto, la efectividad de las metas podría estar relacionada, en cierto modo, con estilo de orientación de meta del deportista.

Otros estudios que han demostrado la efectividad del establecimiento de metas han sido los realizados por Kingston y Hardy (1997), quienes concluyeron que las metas de realización tenían efectos positivos en la auto-eficacia, ansiedad cognitiva y auto-confianza en un grupo de jugadores de golf. Filby, Maynard y Graydon (1999), concluyeron que la combinación de los tres tipos de meta (resultado, realización y rendimiento) provocaba resultados más satisfactorios que el empleo de una de ellas únicamente. Galvan y Ward (1998), por su parte, obtuvieron resultados favorables en relación a la efectividad de esta estrategia cognitiva con tenistas, mientras que Wang (2004) también encontró lo propio en una clase de educación física con una prueba de podómetro electrónico.

Más recientemente, Senécal, Loughead y Gordon (2008), investigaron la influencia que el establecimiento de objetivos grupal tendría en la percepción de cohesión de grupo en jugadoras de baloncesto. A través del empleo del Group Environment Questionnaire (GEQ, Carron, Brawley y Widmeyer, 2002), encontraron diferencias significativas que evidenciaban la efectividad del establecimiento de metas en la percepción de cohesión de grupo de la condición experimental, en relación a la condición control. Otros trabajos como el de Wilson y Brookfield (2009), también han puesto de manifiesto la efectividad del establecimiento de metas de proceso en el ámbito recreacional, incrementando la adherencia y motivación hacia el ejercicio físico.

Novedosas aportaciones en relación a la efectividad del establecimiento de metas, como la de Bueno et al.,(2009), encontraron es su trabajo que los mecanismos motivacionales y afectivos suponen mediadores importantes en la eficacia de esta estrategia para el rendimiento en la resistencia dinámica. Para los autores, el establecimiento de metas es necesario aunque no suficiente para un complejo mecanismo de autoregulación, que incluye, por ejemplo, al esfuerzo y la persistencia. Por su parte, Ortín, Garcés de los Fayos, Gosálvez, Ortega y Olmedilla (2011) han señalado recientemente la interesante conexión entre una orientación optimista del deportista, y su afrontamiento para alcanzar los retos. En ese sentido, la retroalimentación presentada por el entrenador y el tipo de orientación hacia la tarea de los nadadores de su estudio (pesimista u optimista), mediaron en la efectividad de la consecución de la meta. Adicionalmente, Garcés de los Fayos, Olmedilla y Jara (2006) o Pelegrín y Jara (2009) han puesto de manifiesto el valor de la técnica de Programación Neurolingüística (PNL) en relación a la variable motivacional-afectiva en el deporte. En ese sentido, parece que la experiencia subjetiva del deportista y sus representaciones mentales le permitirían activar o generar su propio éxito, especialmente, ante situaciones difíciles. Sin duda, una línea de trabajo que puede arrojar luz al interesante ámbito de estudio de la relación deportista-consecución de metas.

Finalmente, otras revisiones sobre la efectividad de las metas han continuado apoyando la premisa original de Locke (1966, 1968), como en el caso de Burton et al., (2001) quienes en el 78\% de los 56 estudios revisados en el ámbito de la actividad física y el deporte, encontraron efectos positivos moderados a fuertes en esta práctica. Por lo que, al contrario de lo encontrado en aspectos anteriores como la dificultad o flexibilidad de la tarea, aquí sí parecen encontrarse resultados más concluyentes a favor de la efectividad del empleo del establecimiento de metas.

\section{Conclusiones}

Se puede concluir a partir de esta revisión, que el establecimiento de metas parece una herramienta útil para fomentar el rendimiento deportivo en general, así como para la mejora de rendimiento en diversas tareas motoras. Sin embargo, la consistencia aportada por las investigaciones científicas en el ámbito organizacional e industrial (Locke y cols. 1981), no parece tener la misma solidez en el contexto que nos ocupa. En cuanto a la especificidad de las metas y, aunque es conocida la importancia de establecer metas específicas, los resultados derivados de las investigaciones a este respecto no parecen ser concluyentes (Hall et al.,1983; Stitcher et al.,1983). De ese modo, consideramos apropiado manifestar con cautela que las metas específicas, en comparación a las de -hazlo lo mejor que puedas-, parecen ser más efectivas para producir cambios comportamentales en el deportista, con lo que la aplicación de este tipo de metas puede favorecer la mejora del rendimiento modificando la conducta a través de una mejor disposición y orientación hacia los entrenamientos y competiciones.

En relación a la dificultad de la meta, parece existir un mayor beneficio para el rendimiento deportivo cuando se utilizan metas moderadamente difíciles aunque realistas (Vealey, 2007; Kyllo y Landers, 1995; Orlick 2000). No obstante, sería oportuno tener en consideración y profundizar 
en las posibilidades que ofrece lo postulado por Garland (1985) en su Teoría de la Mediación Cognitiva, pues significa otra vía de aproximación al comportamiento de esta variable en la actividad física y el rendimiento deportivo. Si los individuos desarrollan expectativas subjetivas para alcanzar diversos niveles diferentes de ejecución (expectativa de ejecución) y no únicamente el nivel representado por una meta en la (valencia de ejecución), entonces la percepción subjetiva del individuo a cerca de la representación de una meta objetiva, debería jugar un papel crucial en el establecimiento de objetivos para el mismo. Este elemento sugiere que el deportista debe estar completamente involucrado (conjuntamente con el entrenador) en el proceso de establecimiento de metas de distinto tipo (corto, medio, largo plazo; de proceso, resultado, rendimiento; específicos y generales). Por otro lado, es recomendable mantenerse alerta ante situaciones en las que metas demasiado difíciles, o percibidas por el deportista como amenazantes, provoquen estados de indefensión en el mismo (Bueno et al., 2009). Lo encontrado por los autores parece augurar futuras investigaciones entre la dificultad de las metas, en relación a cómo perciben los deportistas las mismas.

En el apartado de la proximidad de la meta, parecen no poder establecerse afirmaciones concluyentes. Algunos autores han insistido en que centrarse únicamente en metas a largo plazo no mejora el rendimiento deportivo (Kyllo y Landers, 1995), o que ambas, metas a corto y a largo plazo, son importantes cara a la consecución de altos logros deportivos (Kane et al., 2001). Parece más adecuado aplicar distintos tipos de metas (corto, medio y largo plazo) puesto que pue-

\section{Referencias}

Bandura, A. (1977). Social learning theory. Englewood Cliffs, NJ: PrenticeHall.

Bandura, A. (1982). Self-efficacy mechanism in human agency. American Psychologist, 37, 122-147.

Bar-Eli, M., Tenenbaum, G., Pie, J. S., Joan S., Btesh, Y. y Almog, A. (2007). Effect of goal difficulty, goal specificity and duration of practice time intervals on muscular endurance performance. En D. Smith, M. Bar-Eli (Eds), Essential readings in sport and exercise psychology. (pp. 305-313). Champaign, ILL: Human Kinetics.

Barnett, M. L. (1977). Effects of two methods of goals setting on learning a gross motor task. Research Quarterly, 48 (1), 19-23.

Barnett, M. L. y Stanicek, J. A. (1979). Effects of goals setting on achievement in archery. Research Quarterly, 50 (3), 328-332.

Bell, K. F. (1983). Championship thinking: The athlete's guide to winning performance in all sports. Englewood Cliffs, NJ: Prentice-Hall.

Botterill, C. (1977). Goal setting and performance on an endurance task. $A$ paper presented at the Canadian Association of Sports Sciences Conference, Winnipeg, Manitoba, Canada.

Botterill, C. (1979). Goal setting with athletes. Sport Science Periodical on Research and Technology in Sport, 1, 1-8.

Boyce, B.A. (1992). The effects of goal proximity on skill acquisition and retention of a shooting task in a field-based setting. Journal of Sport and Exercise Psychology, 14(3), 298-308.

Boyce, B. A. (1994). The effects of goals setting on performance and spontaneous goals-setting behavior of experienced pistol shooters. The Sport Psychologist, 16 (1), 87-93.

Bueno, J., Weinberg, R. S., Fernández-Castro, J. y Capdevilla, L. (2009). Emotional and motivational mechanisms mediating the influence of den ayudar a organizar más específicamente los entrenamientos y las competiciones. Simultáneamente, se fomentaría una mejor orientación en la dirección hacia donde debe dirigirse el deportista (Dosil, 2004).

Estas reflexiones manifiestan la relevancia de esta estrategia, a pesar de que los resultados en el contexto del ejercicio físico y el deporte no hayan sido definitivos. De un modo más consistente a lo encontrado en aspectos comentados anteriormente como la proximidad, dificultad o especificidad en el establecimiento de metas para una tarea motora o gesto deportivo, sí parecen encontrarse resultados más irrebatibles a favor de la efectividad del empleo de esta estrategia. En ese sentido, el establecimiento de metas puede ser más eficaz si además está sujeto a la interacción de mecanismos que actúan como mediadores para su efectividad, como serían la motivación y las emociones (Bueno et al., 2009), un afrontamiento de orientación optimista (Ortín et al., 2011), o las posibilidades que presenta la perspectiva de la programación neurolingüística (Pelegrín y Jara, 2009). Desde la Piscología del Deporte, asimismo, el establecimiento de objetivos debe suponer una herramienta que puede ir estrechamente ligada a, por ejemplo, a la planificación de los ciclos de entrenamiento fisiológico. Sin embargo, no debe olvidarse que las sensaciones, estados de ánimo, períodos alternativos de carga y recuperación, junto con la posible presión añadida de ejecutar ciertos gestos a la perfección, influyen directamente en los resultados y progreso del rendimiento deportivo. Esto demanda del psicólogo deportivo un conocimiento global de la situación en que se halla inmerso el deportista y trabajar en estrecha cooperación con los entrenadores deportivos. goal setting on athletes' performance. Psychology of Sport and Exercise,9(6), 786-800.

Burton, D. (1983). Evaluation of goal setting training on selected cognitions and performance of collegiate swimmers. Unpublished doctoral dissertation, University of Illinois, Urbana.

Burton, D. (1984). Goal setting: A secret to success. Swimming world, 25-29.

Burton, D. (1989). Winning isn't everything: Examining the impact of performance goals on collegiate swimmers' cognitions and performance. Sport Psychologist, 3, 105-132.

Burton, D., Naylor, S. y Holliday, B. (2001). Goal setting in sport: Investigating the goal effectiveness paradigm. En R. Singer, H. Hausenblas, $y$ C. Janelle (Eds.), Handbook of sport psychology (2nd edition, pp. 497-528) New York: Wiley.

Carron, A. V. (1984). Motivation: Implications for coaching and teaching. London, Ontario, Canada: Sports Dynamics.

Carron, A.V., Brawley, L.R., y Widmeyer, W.N. (2002). The Group Environment Questionnaire. Test manual. Morgantown, WV, Fitness Information Technology.

Carver, C. S. y Scheier, M. F. (1982). Control theory: A useful conceptual framework for personality, social, clinical, and health psychology. Psychological Bulletin, 92, 111-135.

Correa, U. C., de Souza, O. P. y Santos, S. (2006). Goal Setting in acquisition of volleyball skill late in motor learning. Perceptual and Motor Skill, 103(1), 273-278.

Cox, R. H. (2002). Sport Psychology: concepts and applications. Boston: McGrawHill.

Davis, J. O. y Spennewyn, K. C. (1983). Goal setting and athletic success En, Hall, E. R. y McIntyre, M. M. (Eds.), Olympism: a movement of the peo- 
ple. United States Olympic Academy VII, (6)3. Texas Tech. University, S.L.

Díaz-Ocejo, J. (2006). Análisis de las Estrategias Cognitivas en Algunos Deportes Individuales y de Adversario. Tesis Doctoral, realizada bajo la Dirección del Dr. Juan Antonio Mora Mérida. Universidad de Málaga: RIUMA.

Dosil, J. (2004). Psicología de la actividad física y del deporte. Madrid: McGrawHill.

Filby, W. C., Maynard, I. W. y Graydon, J. K. (1999). The effect of multiple goal strategies on performance outcomes in training and competition. Journal of Applied Sport Psychology, 11, 230-246.

Galvan, Z. J. y Ward, P. (1998). Effects of public posting on inappropriate on court behaviors by collegiate tennis players. The Sport Psychologist, 12, 419-426.

Gano-Overway, L. A. (2008). The effect of Goal Involvement on SelfRegulatory Processes. International Journal of Sport and exercise Psychology, 6(2), 132-156.

Garcés de los Fayos, Olmedilla, A. y Jara, P. (2006). Psicología y Deporte. Murcia: Diego Marín.

Garland, H. (1983). Influence of ability, assigned goals and normative information on personal goal and performance: A challenge to the goal attainability assumption. Journal of Applied Psychology, 68, 20-30.

Garland, H. (1985). A cognitive mediation theory of task goals and human performance. Motivation and Emotion, 9, 345-367.

Garland, H., Weinberg, R., Bruya, L. y Jackson, A. (1988). Self-efficacy and endurance performance: A longitudinal field test of cognitive mediation theory. Applied Psychology: An international review, 34, 381-394.

González, J. y Garcés de los Fayos, E. (2009). Plan de Entrenamiento Psicológico en el Deporte de la Petanca: En búsqueda del Rendimiento Grupal Óptimo. Revista de Psicología del Deporte, 18, 87-104.

Gould, D. (1991). Establecimiento de metas para el máximo rendimiento. En J.M. Williams (Ed.), Psicología Aplicada al Deporte. Madrid: Biblioteca Nueva.

Hall, H. K, Weinberg, R. y Jackson, A. (1983). The effects of goal setting upon the performance of a circuit training task. Paper presented at TAHPERD Conference, Corpus Christi, TX.

Hall, H. K. y Byrne, T. (1988). Goal setting in sport: Clarifying anomalies. Journal of Sport and Exercise Psychology, 10, 189-192.

Hall, H. K. y Kerr, A. W. (2001). Goal setting in sport and physical activity: Tracing empirical developments and establishing conceptual direction. En G.C. Robert (Ed.), Advances in motivation in sport and exercise (pp.183233).Champaign, IL: Human Kinetics.

Hardy, L., Jones, G. y Gould, D. (1996). Understanding psychological preparation for sport: theory and practice of elite performers. Chichester, UK: John Wiley and Sons.

Hernández-Mendo, A. y Ramos-Pollán, R. (1995). Aplicación informática para evaluación y entrenamiento de la atención en psicología del deporte. Psicothema, 7, 527-529.

Hollingsworth, B. (1975). Effects of performance goals and anxiety on learning a gross motor task. Research Quarterly, 46 (2), 162-168.

Jackson, S. y Csikszentmihalyi, M. (2002). Fluir en el deporte: Claves para experiencias y situaciones óptimas. Paidotribo, Barcelona.

Johnson, S. R., Morgantown, W., Ostrow, A. C., Perna, F. M. y Etzel, E. F. (1997). The effects of group versus individual goal setting on bowling performance. Sport Psychologist, 11(2), 190-200.

Kane, T., Baltes, T. y Moss, M. (2001). Causes and consequences of free-set goal: An investigation of athletic self-regulation. Journal of Sport and Exercise Psychology, 23, 55-75.

Kenan, M. C. y Lord, R. G. (1988). Effects of participative vs. assigned goals and feedback in a multitrial task. Motivation and Emotion, 12, 75-86.

Kingston, K. M. y Hardy, L. (1997). Effects of different types of goals on processes that support performance, The Sport Psychologist, 11, 277-293.

Kirschenbaum, D. S. (1985). Proximity and specifity of planning: A position paper. Cognitive Therapy and Research, 9, 489-506.

Kyllo, L. B. y Landers, D. M. (1995). Goal setting in sport and exercise: A research synthesis to resolve the controversy. Journal of Sport and Exercise Psychology, 17, 117-137.

Locke, E. A. (1966). The relationships of intentions to level of performance. Journal of Applied Psychology, 50, 60-66.

Locke, E. A. (1968). Toward a theory of task motivation incentives. Journal of Organizational Behavior and Human Performance, 3, 157-189.
Locke, E. A. y Bryan, J. F. (1966). Cognitive aspects of psychomotor performance: The effects of performance goals on levels of performance. Journal of Applied Psychology, 50 (4), 286-291.

Locke, E. A. y Latham, G. P. (1985). Goal setting in sport. Journal of Sport Psychology, 7, 205- 222.

Locke, E. A., Shaw, K. N., Saari, L. M. y Latham, G. P. (1981). Goal setting and task performance. Psychological Bulletin, 90, 125-152.

Martens, R., Christina, R. W., Harvey, J. S., Jr. y Sharkey, B. J. (1981). Coaching young atbletes. Champaign, Ill.: Human Kinetics.

McClements, J. (1982). Goal setting and planning for mental preparations. En L. Wankel y R. B. Wilberg (Eds.), Psychology of sport and motor behavior: Research and practice. Proceedings of the Annual Conference of the Canadian Society for Psychomotor Learning and Sport Psychology. Edmonton, Alberta, Canada: University of Alberta.

Mento, A. J., Locke, E. y Klein, H. (1992). Relationship of goal level to valence and instrumentality. Journal of Applied Psychology, 77, 395-405.

Mora, J. A., García, J., Toro, S. y Zarco, J. A. (2000). Psicología Aplicada a la Actividad Fisico-Deportiva. Madrid: Pirámide.

Moreno, J.A., Cervelló, E. y González, D. (2010). La teoría de las metas de logro y la teoría de la autodeterminación como predictores del flow disposicional en jóvenes deportistas. Anales de Psicología, 26(2), 102-122.

O'Block, F. R. y Evans, F. H. (1984). Goal setting as a motivational technique. En J. M. Silva y R. S. Weinberg (Eds.), Psychological Foundations of Sports. Champaign, Ill.: Human Kinetics.

Olmedilla, A. Ortega, E., Almeida, P., Lameiras, J., Villalonga, T., Sousa, C., Torregrosa, M, Cruz, J. y García-Mas, A. (2011). Cohesión y cooperación en Equipos Deportivos. Anales de Psicología, 27(1), 151-178.

Orlick, T. (2000). In pursuit of excellence: How to win in sport and life through mental training. (3rd edition). Champaign, Ill.: Human Kinetics.

Ortín, F. J., Garcés de los Fayos, E. J., Gosálvez, J., Ortega, E. y Olmedilla, A. (2011).Optimismo y ejecución en el deporte en situaciones adversas. Replicando a Seligman 1990. Revista de Psicología del Deporte, 20 (2), 491501.

Pelegrín, A. y Jara, P. (2009). Manipulación de submodalidades de la representación mental en el trabajo psicológico con taekwondistas: una intervención en el marco de la PNL. Cuadernos de Psicología del Deporte, 9, 3951.

Pierce, B. P. y Burton, D. (1998). Scoring the perfect 10: Investigating the impact of goal-setting styles on a goal-setting program for female gymnasts. Sport Psychologist, 12(2), 156-168.

Pinder, C. (1984). Working motivation. Glenview, Il: Scott, Foresman.

Riera, J. (1994). Aprendizaje de la táctica deportiva. Revista de Psicología del Deporte, 5, 111-124.

Riera, J. (1997). Acerca del deporte y del deportista. Revista de Psicología del Deporte, 11, 127-136.

Ryan, T. A. (1970). Intentional behavior: An approach to human motivation. Ronald Press: New York.

Senecal, J., Todd, M. L. y Gordon, A. B. (2008). A season-long teambuilding intervention: Examining the effect of team goal setting on cohesion. Journal of Sport and Exercise Psychology, 30, 186-199.

Sicilia, A., Aguila, C., Muyor, J.M. , Orta, A. y Moreno, J.A. (2009). Perfiles motivacionales de los usuarios en Centros Deportivos Municipales. Anales de Psicología, 25(1), 175-190.

Stitcher, T., Weinberg, R. y Jackson, A. (1983). Goal setting and its effects on endurance performance. Paper presented at the TAHPERD Conference, Corpus Christi, TX.

Taylor, F. W. (1967). The Principles of Scientific Management. Test manual. Morgantown, WV: Fitness Information Technology. New York: Norton and Company.

Vealey, R. S. (2007). Mental Skills Training in Sport. En Tenenbaum, G. y Eklund, R. C. (Eds.), Handbook of Sport Psychology, $3^{\text {rd }}$ edition (pp.287309).New York: Wiley.

Wang, S. H. (2004). The effects of goal setting on physical activity of female middle school students. Doctoral dissertation, Florida State University, Talahasse, FL.

Weinberg R. S. y Gould D. (2003). Foundations of Sport And Exercise Psychology. Champaign, Il: Human Kinetics.

Weinberg, R. S. (1995). Establecimiento de metas y ejecución motora: Análisis y crítica. En G. C. Roberts (Ed.), Motivación en el deporte y el ejercicio. Bilbao: Desclée De Brouwer. 
Weinberg, R. S., Bruya, L. D. y Jackson, A. (1985). The effects of goal proximity and goal specifity on endurance performance. Journal of Sport Psychology, 7, 296-305.

Weinberg, R. S., Bruya, L. D., Jackson, A. y Garland, H. (1987). Goal difficulty and endurance performance: A challenge to the goal attainability assumption. Journal of Sport Behavior, 10, 82-92.

Weinberg, R. S., Burke, K. L. y Jackson, A. (1997). Coaches' and players' perceptions of goal setting in junior tennis: An exploratory investigation. Sport Psychologist, 11(4), 426-429.
Weinberg, R. S., Burton, D., Yukelson, D. y Weigand, D. (1993). Goal setting in competitive sport: An exploratory investigation of practices of collegiate athletes. Sport Psychologist, 7(3), 275-289.

Weinberg, R. S., Burton, D., Yukelson, D. y Weigand, D. (2000). Perceived goal setting practices of Olympic athletes: An exploratory investigation. The Sport Psychologist, 14, 279-295.

Wilson, K. y Brookfield, D. (2009). Effect on goal setting on motivation and adherence in a six-week exercise program. International Journal of Sport and Exercise Psychology, 6, 89-100.

(Artículo recibido: 03-10-2011, revisión: 25-10-2011, aceptado: 02-12-2011) 